PROCEEDINGS OF THE

AMERICAN MATHEMATICAL SOCIETY

Volume 131, Number 1, Pages 175-183

S 0002-9939(02)06509-7

Article electronically published on May 22, 2002

\title{
DIFFERENTIAL EQUATIONS OVER POLYNOMIALLY BOUNDED O-MINIMAL STRUCTURES
}

\author{
JEAN-MARIE LION, CHRIS MILLER, AND PATRICK SPEISSEGGER
}

(Communicated by Carl G. Jockusch, Jr.)

\begin{abstract}
We investigate the asymptotic behavior at $+\infty$ of non-oscillatory solutions to differential equations $y^{\prime}=G(t, y), t>a$, where $G: \mathbb{R}^{1+l} \rightarrow \mathbb{R}^{l}$ is definable in a polynomially bounded o-minimal structure. In particular, we show that the Pfaffian closure of a polynomially bounded o-minimal structure on the real field is levelled.
\end{abstract}

A classical topic in asymptotic analysis is the study of the behavior of solutions at $+\infty$ to equations $P\left(t, y(t), \ldots, y^{(n)}(t)\right)=0$, where $P: \mathbb{R}^{2+n} \rightarrow \mathbb{R}$ is a nontrivial polynomial function. It was conjectured by $E$. Borel that the growth of such solutions should be bounded at $+\infty$ by the $(n+1)$-st compositional iterate of $e^{x}$. This turned out to be false - see Boshernitzan [2 for a detailed account-unless infinitely oscillating solutions are somehow ruled out. But with such assumptions, sharper estimates are obtained that rule out transexponential growth as well as certain kinds of intermediate asymptotic behavior (e.g., that of solutions to the functional equation $g \circ g=e^{x}$; see Rosenlicht [23, §2]). In this paper, we extend these results to (systems of) first-order ODEs defined over polynomially bounded o-minimal structures on the real field. Examples of large classes of maps that generate such structures are given by van den Dries and Speissegger [9, 10 and Rolin et al. 20.

Some of our results hold in o-minimal expansions of arbitrary ordered fields, while some appear to make sense only over the real numbers. All but one we prove by so-called standard methods (that is, without model theory). We organize the exposition as follows: First, results are stated over $\mathbb{R}$ in the form of an extended abstract, followed by proofs. Then we indicate those results that, after appropriate modification, hold in o-minimal expansions of arbitrary ordered fields.

Conventions. The reader is assumed to be familiar with the basic properties of o-minimal structures on $(\mathbb{R},+, \cdot)$; see e.g. van den Dries and Miller $[8, \S \S 2,4]$. From now on, unless stated otherwise, "structure" abbreviates "structure on $(\mathbb{R},+, \cdot)$ ", and "definable" means "definable with parameters" in the structure under consideration.

Received by the editors April 11, 2000 and, in revised form, August 20, 2001.

2000 Mathematics Subject Classification. Primary 26A12, 34E99; Secondary 34E05, 03C64.

The second author's research was supported by NSF Grants DMS-9896225 and DMS-9988855.

The third author's research was supported in part by NSERC Grant OGP0009070. 
Definition. Let $\mathfrak{R}$ be a structure and $m \in \mathbb{N}$. A map $\gamma:(a, \infty) \rightarrow \mathbb{R}^{m}$ is nonoscillatory over $\mathfrak{R}$ if for each definable function $f: \mathbb{R}^{1+m} \rightarrow \mathbb{R}$ there exists $t_{f} \geq a$ such that either $f(t, \gamma(t))=0$ for all $t>t_{f}$ or $f(t, \gamma(t)) \neq 0$ for all $t>t_{f}$.

Note. The definition implies that $\mathfrak{R}$ is o-minimal. To see this, let $A \subseteq \mathbb{R}$ be definable. The non-oscillation condition applied to the characteristic function of $A \times \mathbb{R}^{m}$ yields that $A$ either is bounded or contains some open ray $(c, \infty)$. It follows that the boundary of every definable subset of $\mathbb{R}$ is finite (see van den Dries et al. 7, Lemma 5.2] for details) so $\mathfrak{R}$ is o-minimal.

Examples. (1) If $\mathfrak{R}^{\prime}$ is an o-minimal expansion of $\mathfrak{R}$, then every map $(a, \infty) \rightarrow \mathbb{R}^{m}$ definable in $\mathfrak{R}^{\prime}$ is non-oscillatory over $\mathfrak{R}$.

(2) The function $e^{x}+\sin x$ is non-oscillatory over every polynomially bounded o-minimal structure (this is an easy consequence of cell decomposition and the definition of "polynomially bounded"). Moreover, since it is a solution on $\mathbb{R}$ to $y^{(4)}=y$, it is a component function of a first-order ODE defined over $(\mathbb{R},+, \cdot)$. However, we shall see that it is not a component function of any non-oscillatory solution to any first-order ODE over any o-minimal structure (see the remarks following the proof of Lemma 1).

(3) Let $\xi: \mathbb{R}^{3} \rightarrow \mathbb{R}^{2}$ be defined by

$$
\left(t, y_{1}, y_{2}\right) \mapsto \begin{cases}\left(-\frac{1}{t}-y_{1}+y_{2},-y_{1}-y_{2}\right) & \text { if } t \neq 0, \\ (0,0) & \text { if } t=0 .\end{cases}
$$

(Note that $\xi$ may be regarded as a small (at $t=+\infty$ ) perturbation of the plane vector field $\dot{\mathbf{x}}=\left(\begin{array}{cc}-1 & 1 \\ -1 & -1\end{array}\right) \mathbf{x}$, all of whose non-trivial trajectories spiral infinitely toward the origin.) It follows from work of Cano et al. (both published 3 and ongoing) that each of the infinitely many solutions $\gamma$ on $(0, \infty)$ to $y^{\prime}=\xi(t, y)$ is non-oscillatory over $\mathbb{R}_{\mathrm{an}}$, the expansion of $(\mathbb{R},+, \cdot)$ by all restricted analytic functions. It is not known if $(\mathbb{R},+, \cdot, \gamma)$ is o-minimal for any such $\gamma$. This example, and others of like kind, motivated the definition of "non-oscillatory over". See Corollary [3] and its proof for more information.

Unary (that is, $\mathbb{R} \rightarrow \mathbb{R}$ ) functions $f$ and $g$ are said to have the same germ (at $+\infty$ ) if ultimately $f=g$. (Ultimately abbreviates "for all sufficiently large positive arguments".) We do not distinguish notationally between a function and its germ. Let $x$ denote the germ of the identity map on $\mathbb{R}$, and identify the germ of an ultimately constant function with the corresponding real number.

A unary function $f$ is infinitely increasing if there exists $a \in \mathbb{R}$ such that $f$ is strictly increasing and unbounded above on $(a, \infty)$. A germ is infinitely increasing if it is the germ of an infinitely increasing function. Let $\mathcal{I}$ denote the set of all infinitely increasing germs.

Put $e_{0}(t)=t$ and $e_{n+1}(t)=\exp \left(e_{n}(t)\right)$ for $n \in \mathbb{N}$ and $t \in \mathbb{R}$. Similarly, put $\ell_{0}(t)=t$ and $\ell_{n+1}(t)=\log \left(\ell_{n}(t)\right)$ for $n \in \mathbb{N}$ and $t \in \mathbb{R}$. (For convenience, we set $\log (t)=0$ for $t \leq 0$, so that each function $\ell_{n}$ is defined on all of $\mathbb{R}$.) We may also write $\ell_{-n}$ for $e_{n}$ and $e_{-n}$ for $\ell_{n}$, depending on convenience (for example, $\ell_{j+k}=\ell_{j} \circ \ell_{k}$ for all $j, k \in \mathbb{Z}$ ).

We write $f \sim g$ if $g$ ultimately has no zeros and $\lim _{t \rightarrow+\infty}[f(t) / g(t)]=1$.

A function (or germ) $u$ has level if $u \in \mathcal{I}$ and there are $k, s \in \mathbb{Z}$ with $\ell_{k+s} \circ u \sim$ $\ell_{k}$; we then say that $u$ has level $s$ and write level $(u)=s$. Basic properties of level can be found in [23]. See Shackell [24] for a detailed examination (from a 
somewhat different point of view) and Marker and Miller [14 for some modeltheoretic considerations.

We now fix $l \geq 1, G: \mathbb{R}^{1+l} \rightarrow \mathbb{R}^{l}$ and $F=\left(F_{1}, \ldots, F_{l}\right): \mathbb{R} \rightarrow \mathbb{R}^{l}$ such that ultimately $F^{\prime}(t)=G(t, F(t))$. Let $\mathfrak{R}_{G}$ denote the structure $(\mathbb{R},+, \cdot, G)$.

Given $h: \mathbb{R}^{1+l} \rightarrow \mathbb{R}, h(x, F)$ denotes the germ of $t \mapsto h(t, F(t))$. Let $\mathcal{H}$ be the set of all germs of unary functions definable in $\mathfrak{R}_{G}$, and $\mathcal{H}\langle\langle F\rangle\rangle$ be the set of all germs $h(x, F)$ where $h: \mathbb{R}^{1+l} \rightarrow \mathbb{R}$ is definable in $\mathfrak{R}_{G}$.

Theorem 1. Let $\mathfrak{R}_{G}$ be polynomially bounded, $F$ be non-oscillatory over $\mathfrak{R}_{G}$, and $f \in \mathcal{H}\langle\langle F\rangle\rangle$. Then exactly one of the following holds:

- $f \in \mathbb{R}$.

- $|f|$ is infinitely increasing and has level bounded in absolute value by $l$.

- There exists $c \in \mathbb{R}$ such that $1 /|f-c|$ is infinitely increasing and has level bounded in absolute value by $l$.

Remarks.

- In particular, $F_{i}(t)=o\left(e_{l+1}(t)\right)$ as $t \rightarrow+\infty, i=1, \ldots, l$.

- Bounding the absolute values of the levels by $l$ is sharp. Consider (a) $F_{i}=e_{i}$ for $i=1, \ldots, l$; (b) $F_{i}=\ell_{i}$ for $i=1, \ldots, l$.

- The assumption that $\mathfrak{R}_{G}$ be polynomially bounded is necessary. (If not, by growth dichotomy [16], $e^{x}$ is definable, hence so is $e_{2}(x)$. Then $e_{2} \in \mathcal{H}$, level $\left(e_{2}\right)=2$, and $e_{2}^{\prime}=G\left(x, e_{2}\right)$, where $G(t, y)=e^{t} y$.)

Corollary 1. Let $n \in \mathbb{N}, f$ be a $C^{n}$ germ, and $g: \mathbb{R}^{2+n} \rightarrow \mathbb{R}$ be such that $g^{-1}\{0\}$ has no interior and $g\left(x, f, \ldots, f^{(n)}\right)=0$. If $(\mathbb{R},+, \cdot, g)$ is polynomially bounded and $\left(f, \ldots, f^{(n)}\right)$ is non-oscillatory over $(\mathbb{R},+, \cdot, g)$, then either $f \in \mathbb{R}$, or $|\operatorname{level}(|f|)| \leq$ $n$, or there exists $c \in \mathbb{R}$ such that $|\operatorname{level}(1 /|f-c|)| \leq n$.

In polynomially bounded o-minimal structures, for each $m \in \mathbb{N}$, the ring of all $C^{\infty}$ definable functions $\mathbb{R}^{m} \rightarrow \mathbb{R}$ is an integral domain [17]. Hence, in the above, if $g$ is $C^{\infty}$ and nontrivial, then the assumption that $g^{-1}\{0\}$ have no interior is superfluous, and we have a natural generalization of the case that $g$ is a polynomial function.

Given $A \subseteq \mathbb{R}^{l}$, let $\mathrm{d}(p, A)$ denote the Euclidean distance of $p \in \mathbb{R}^{l}$ to $A$.

Corollary 2. Let $\mathfrak{R}_{G}$ be polynomially bounded, $F$ be non-oscillatory over $\mathfrak{R}_{G}$, and $A \subseteq \mathbb{R}^{l}$ be definable in $\mathfrak{R}_{G}$. Suppose that $\mathrm{d}(F(t), A) \rightarrow 0$ as $t \rightarrow+\infty$. Then either $F$ ultimately lies in $A$ or there exists $k \in \mathbb{Z}$ such that for each $\epsilon>0$

$$
\frac{1}{e_{k+l}\left((1+\epsilon) \ell_{k}(t)\right)}<\mathrm{d}(F(t), A)<\frac{1}{e_{k-l}\left((1-\epsilon) \ell_{k}(t)\right)} \quad \text { as } t \rightarrow+\infty .
$$

Corollary 3. Let $\xi$ be as in Example 3 and $\gamma:(0, \infty) \rightarrow \mathbb{R}^{2}$ be a solution on $(0, \infty)$ to $y^{\prime}(t)=\xi(t, y(t))$. Let $0 \in A \subseteq \mathbb{R}^{2}$ be subanalytic with empty interior. Then there exists $k \in \mathbb{Z}$ such that for each $\epsilon>0$

$$
\frac{1}{e_{k+2}\left((1+\epsilon) \ell_{k}(t)\right)}<\mathrm{d}(\gamma(t), A)<\frac{1}{e_{k-2}\left((1-\epsilon) \ell_{k}(t)\right)} \quad \text { as } t \rightarrow+\infty .
$$

(See e.g. Bierstone and Milman [1] for information on subanalytic geometry.)

The conclusion of Theorem $\mathbb{1}$ is sharper if $\left(\mathfrak{R}_{G}, F\right)$ - the expansion of $\mathfrak{R}_{G}$ by $F$-is o-minimal. Recall that the field of exponents of $\mathfrak{R}_{G}$ is the set of all $r \in \mathbb{R}$ such that the function $t \mapsto t^{r}:(0, \infty) \rightarrow \mathbb{R}$ is definable. 
Theorem 2 (cf. 22]). Suppose that $\left(\mathfrak{R}_{G}, F\right)$ is o-minimal and $\mathfrak{R}_{G}$ is polynomially bounded with field of exponents $K$. Then there exist $d \in\{0, \ldots, l\}$ and $0<$ $u_{1}, \ldots, u_{d} \in \mathcal{H}\langle\langle F\rangle\rangle \backslash \mathcal{H}$ such that for each $f \in \mathcal{H}\langle\langle F\rangle\rangle \backslash\{0\}$ there exist $c \neq 0$ and $r_{0}, \ldots, r_{d} \in K$ with $f \sim c x^{r_{0}} u_{1}^{r_{1}} \cdots u_{d}^{r_{d}}$.

(We don't know if the conclusion above holds if $F$ is assumed only to be nonoscillatory over $\mathfrak{R}_{G}$.)

Let $\mathfrak{R}$ be a structure. The Pfaffian closure of $\mathfrak{R}$ is the smallest expansion of $\mathfrak{R}$ that is closed under taking Rolle leaves of definable $C^{1} 1$-forms. If $\mathfrak{R}$ is o-minimal, then so is its Pfaffian closure. (See 25] for details and complete definitions.)

Theorem 3. If $\mathfrak{R}$ is polynomially bounded and o-minimal, then its Pfaffian closure is levelled.

(A structure is levelled if every infinitely increasing definable function has level.)

As of this writing, every structure known to be o-minimal is a reduct (in the sense of definability) of the Pfaffian closure of some polynomially bounded o-minimal structure. Hence, every structure presently known to be o-minimal is levelled.

\section{ProOfs}

We work in the setting of Hardy fields. We assume the reader to be familiar with [21, $\S \S 1-3]$ and [23, $\S \S 1,2]$. For the most part, we use the same notation, terminology and conventions. We also make use of $C^{1}$ cell decomposition; see 6 . Chapters 3, 7].

Lemma 1. Let $F$ be non-oscillatory over $\mathfrak{R}_{G}$. Then $\mathcal{H}\langle\langle F\rangle\rangle$ is a Hardy field.

Proof. That $\mathcal{H}\langle\langle F\rangle\rangle$ is a ring is clear. By non-oscillation, it is an integral domain. Let $f \in \mathcal{H}\langle\langle F\rangle\rangle$; then $f=h(x, F)$ where $h: \mathbb{R}^{1+l} \rightarrow \mathbb{R}$ is definable in $\mathfrak{R}_{G}$. If $f \neq 0$, then $1 / f=g(x, F)$, where $g: \mathbb{R}^{1+l} \rightarrow \mathbb{R}$ is defined by

$$
g(t, y)= \begin{cases}1 / h(t, y), & h(t, y) \neq 0 \\ 1, & \text { otherwise }\end{cases}
$$

Hence, $\mathcal{H}\langle\langle F\rangle\rangle$ is a field. It remains to be shown that $f$ is differentiable and $f^{\prime} \in$ $\mathcal{H}\langle\langle F\rangle\rangle$. Take a decomposition $\mathcal{D}$ of $\mathbb{R}^{1+l}$ into $C^{1}$ cells definable in $\mathfrak{R}_{G}$ such that the restriction $h\left\lceil D\right.$ is $C^{1}$ for each $D \in \mathcal{D}$. Since $F$ is non-oscillatory over $\mathfrak{R}_{G}$, there exists $D \in \mathcal{D}$ such that ultimately $(t, F(t)) \in D$. Put $d=\operatorname{dim} D$; then there is some coordinate projection $\pi: \mathbb{R}^{1+l} \rightarrow \mathbb{R}^{d}$ such that $\pi\lceil D$ maps $D$ diffeomorphically onto an open cell in $\mathbb{R}^{d}$. Let $\phi$ denote the compositional inverse of $\pi\lceil D$. Note that $\phi$ is definable in $\mathfrak{R}_{G}$, hence so is the derivative of $h \circ \phi$. Then

$$
\begin{aligned}
f^{\prime} & =\left((h \circ \phi)^{\prime} \circ \pi(x, F)\right) \cdot(\pi(x, F))^{\prime} \\
& =\left((h \circ \phi)^{\prime} \circ \pi(x, F)\right) \cdot \pi(1, G(x, F)) \in \mathcal{H}\langle\langle F\rangle\rangle .
\end{aligned}
$$

Remarks. (i) The converse of Lemma11holds. (ii) Consider $f:=e^{x}+\sin x$. As noted earlier, $f$ is non-oscillatory over every polynomially bounded o-minimal structure. On the other hand, we have $f-f^{\prime \prime}=2 \sin x$, so $f$ does not belong to any Hardy field. Hence, $f$ is not a component function of any non-oscillatory solution to a first-order ODE over any o-minimal structure. 
Lemma 2. Let $\mathfrak{R}$ be an o-minimal expansion of $(\mathbb{R},<)$ and $f_{1}, \ldots, f_{n}: \mathbb{R} \rightarrow \mathbb{R}$ be arbitrary functions. Suppose there exist $c \in \mathbb{R}$ and a non-open cell $D \subseteq \mathbb{R}^{1+n}$ definable in $\mathfrak{R}$ such that the graph of $t \mapsto\left(f_{1}(t), \ldots, f_{n}(t)\right):(c, \infty) \rightarrow \mathbb{R}^{n}$ is contained in $D$. Then there exist $k \in\{0, \ldots, n-1\}$ and $g: \mathbb{R}^{1+k} \rightarrow \mathbb{R}$ definable in $\mathfrak{R}$ such that, as germs, $f_{k+1}=g\left(x, f_{1}, \ldots, f_{k}\right)$.

Proof. The projection of $D$ on the first coordinate contains $(c, \infty)$. It follows from the definition of cell that there is a maximal integer $k \in\{0, \ldots, n-1\}$ such that the projection $\pi D$ on the first $1+k$ coordinates is an open cell. Then there is a function $g: \mathbb{R}^{1+k} \rightarrow \mathbb{R}$ definable in $\mathfrak{R}$ such that the projection of $D$ on the first $1+k+1$ coordinates is equal to the graph of $g\lceil\pi D$.

Lemma 3. Let $F$ be non-oscillatory over $\mathfrak{R}_{G}$ and $u_{1}, \ldots, u_{l+1} \in \mathcal{H}\langle\langle F\rangle$. Then there exist $k \in\{0, \ldots, l\}$ and $h: \mathbb{R}^{1+k} \rightarrow \mathbb{R}$ definable in $\mathfrak{R}_{G}$ such that $u_{k+1}=$ $h\left(x, u_{1}, \ldots, u_{k}\right)$.

Proof. There exist functions $h_{1}, \ldots, h_{l+1}: \mathbb{R}^{1+l} \rightarrow \mathbb{R}$ definable in $\mathfrak{R}_{G}$ with $u_{i}=$ $h_{i}(x, F)$ for $i=1, \ldots, l+1$. Note that the map $t \mapsto\left(u_{1}(t), \ldots, u_{l+1}(t)\right)$ is nonoscillatory over $\mathfrak{R}_{G}$. Put $y=\left(y_{1}, \ldots, y_{l}\right)$ and let $A$ be the graph of the map

$$
(t, y) \mapsto\left(h_{1}(t, y), \ldots, h_{l+1}(t, y)\right): \mathbb{R}^{1+l} \rightarrow \mathbb{R}^{l+1} .
$$

Let $B \subseteq \mathbb{R}^{1+l+1}$ be the image of $A$ under the projection

$$
(t, y, z) \mapsto(t, z): \mathbb{R}^{1+l+1+l} \rightarrow \mathbb{R}^{1+l+1} .
$$

Since $\operatorname{dim} A=l+1$, we have $\operatorname{dim} B \leq l+1<l+2$. Hence, $B$ is a finite disjoint union of non-open cells definable in $\mathfrak{R}_{G}$, and $\left(t, u_{1}(t), \ldots, u_{l+1}(t)\right)$ is contained ultimately in one of them. Apply Lemma 2

Recall that $u, v \in \mathcal{I}$ are comparable if there exists $N \in \mathbb{N}$ such that $u \leq v^{N}$ and $v \leq u^{N}$. Comparability is an equivalence relation on $\mathcal{I}$. The comparability class $\mathrm{Cl}(u)$ of $u \in \mathcal{I}$ is the equivalence class of $u$ modulo comparability. The rank of a Hardy field $k$ is the number of comparability classes of $k \cap \mathcal{I}$. Note that, by growth dichotomy, $\mathfrak{R}_{G}$ is polynomially bounded if and only if $\mathcal{H}$ has rank 1 .

Lemma 4. Suppose $\mathfrak{R}_{G}$ is polynomially bounded and o-minimal. Let $h: \mathbb{R}^{1+k} \rightarrow \mathbb{R}$ be definable in $\mathfrak{R}_{G}$ and $u_{0}, \ldots, u_{k} \in \mathcal{I}$ be such that $\mathrm{Cl}\left(u_{0}\right)<\mathrm{Cl}\left(u_{1}\right)<\cdots<\mathrm{Cl}\left(u_{k}\right)$. Then $\left|h\left(u_{0}, u_{1}, \ldots, u_{k}\right)\right| \leq u_{k}^{N}$ for some $N \in \mathbb{N}$.

Proof. We proceed by induction on $k \geq 0$.

The case $k=0$ is immediate by polynomial bounds.

Let $k>0$. By [8 C.4], there exist $N \in \mathbb{N}$ and $\rho: \mathbb{R}^{k} \rightarrow(0, \infty)$ definable in $\mathfrak{R}_{G}$ such that $\left|h\left(a_{0}, \ldots, a_{k-1}, t\right)\right| \leq t^{N}$ for all $a_{0}, \ldots, a_{k-1} \in \mathbb{R}$ and $t>\rho\left(a_{0}, \ldots, a_{k-1}\right)$. Inductively, $\rho\left(u_{0}, \ldots, u_{k-1}\right) \leq u_{k-1}^{M}$ for some $M \in \mathbb{N}$. Since $\mathrm{Cl}\left(u_{k-1}\right)<\mathrm{Cl}\left(u_{k}\right)$, we have $u_{k}>\rho\left(u_{0}, \ldots, u_{k-1}\right)$, so $\left|h\left(u_{0}, u_{1}, \ldots, u_{k}\right)\right| \leq u_{k}^{N}$.

Remark. A stronger conclusion holds: Either $h\left(u_{0}, \ldots, u_{k}\right)=0$, or there exist $c \in \mathbb{R} \backslash\{0\}$ and $r_{0}, \ldots, r_{k} \in K$ such that $h\left(u_{0}, \ldots, u_{k}\right) \sim c \prod_{i=0}^{k} u_{i}^{r_{i}}$, where $K$ is the field of exponents of $\mathfrak{R}_{G}$. We do not need this stronger version here, so we leave the proof to the interested reader. (Replace $u_{0}$ by $u_{0}^{-1}$ and reduce to the case that $u_{0}=x$. Then the map $t \mapsto\left(u_{1}(t), \ldots, u_{k}(t)\right)$ is non-oscillatory over $\mathfrak{R}_{G}$. Use [15] Proposition 5.2] instead of [8, C.4].) 
Proof of Theorem 1 . Suppose that $\mathfrak{R}_{G}$ is polynomially bounded and $F$ is non-oscillatory over $\mathfrak{R}_{G}$. It suffices to show that every $u \in \mathcal{I} \cap \mathcal{H}\langle\langle F\rangle\rangle$ has level bounded in absolute value by $l$. By Lemma $1 \mathcal{H}\langle\langle F\rangle\rangle$ is a Hardy field. We claim that it has at most $l$ comparability classes other than $\mathrm{Cl}(x)$. Suppose otherwise; then there exist infinitely increasing $u_{1}, \ldots, u_{l+1} \in \mathcal{H}\langle\langle F\rangle\rangle \backslash \mathcal{H}$ such that $\mathrm{Cl}\left(u_{1}\right)<\cdots<\mathrm{Cl}\left(u_{l+1}\right)$ and $\mathrm{Cl}\left(u_{i}\right) \neq \mathrm{Cl}(x)$ for all $i \in\{1, \ldots, l+1\}$. Reparametrizing by the compositional inverse of $u_{1}$, we may assume that $\mathrm{Cl}(x)<\mathrm{Cl}\left(u_{1}\right)$. By Lemma 3 there exist $k \in\{1, \ldots, l\}$ and $h: \mathbb{R}^{1+k} \rightarrow \mathbb{R}$ definable in $\mathfrak{R}_{G}$ such that $u_{k+1}=h\left(x, u_{1}, \ldots, u_{k}\right)$. Apply Lemma 4 for a contradiction. Hence, $\mathcal{H}\langle\langle F\rangle\rangle$ has rank at most $l+1$. Now, [23, Theorem 1] yields that every $u \in \mathcal{I} \cap \mathcal{H}\langle\langle F\rangle\rangle$ has level bounded in absolute value by $l+1$. But, since $\mathcal{H}$ is a rank 1 Hardy field containing $x$, we may apply 21, Theorem 3] to improve the bound to $l$.

Remark. A converse to Theorem 1 holds: Drop the assumption that $\mathfrak{R}_{G}$ be o-minimal and suppose instead that $\mathcal{H}\langle\langle F\rangle\rangle$ is a finite rank Hardy field. Then $F$ is non-oscillatory over $\mathfrak{R}_{G}$, which in turn implies that $\mathfrak{R}_{G}$ is o-minimal (recall the remark following the definition of "non-oscillatory over"). Since $\mathcal{H}\left\langle\langle F\rangle\right.$ has finite rank, $\mathfrak{R}_{G}$ is not exponential (otherwise $e_{n} \in \mathcal{H}$ for all $n \in \mathbb{N}$ ). By growth dichotomy, $\mathfrak{R}_{G}$ is polynomially bounded.

Proof of Corollary [1] By Lemma 2, there exist $l \leq n$ and $h: \mathbb{R}^{1+l} \rightarrow \mathbb{R}$ definable in $(\mathbb{R},+, \cdot, g)$ such that $f^{(l)}=h\left(x, f, \ldots, f^{(l-1)}\right)$. Rewrite as a system of first-order ODEs and apply Theorem 1

Proof of Corollary 2 Since $A$ is definable in $\mathfrak{R}_{G}$, so is $p \mapsto \mathrm{d}(p, A): \mathbb{R}^{l} \rightarrow \mathbb{R}$. Then the germ $\mathrm{d}(F, A)$ of $t \mapsto \mathrm{d}(F(t), A)$ is an element of $\mathcal{H}\langle\langle F\rangle$. By assumption, we have $\nu(\mathrm{d}(F, A))>0$, so either $\mathrm{d}(F, A)=0$ or $1 / \mathrm{d}(F, A) \in \mathcal{I}$. If the former, then $F$ ultimately lies in $A$, so assume the latter. By Theorem 1 there are integers $k, s$ such that $|s| \leq l$ and $\ell_{k+s}(1 / \mathrm{d}(F, A)) \sim \ell_{k}$. Fix $\epsilon>0$. Then

$$
(1-\epsilon) \ell_{k}<\ell_{k+s}(1 / \mathrm{d}(F, A))<(1+\epsilon) \ell_{k}
$$

and so

$$
e_{k+s}\left((1-\epsilon) \ell_{k}\right)<1 / \mathrm{d}(F, A)<e_{k+s}\left((1+\epsilon) \ell_{k}\right) .
$$

Since $|s| \leq l$, we have

$$
\frac{1}{e_{k+l}\left((1-\epsilon) \ell_{k}(t)\right)}<\mathrm{d}(F(t), A)<\frac{1}{e_{k-l}\left((1+\epsilon) \ell_{k}(t)\right)} \quad \text { as } t \rightarrow+\infty .
$$

Proof of Corollary [3] We provide only a sketch. Familiarity with [4] is assumed.

First, it is routine to obtain an explicit formal solution

$$
\Gamma(T):=\left(\sum_{n=1}^{\infty} a_{n} T^{-n}, \sum_{n=1}^{\infty} b_{n} T^{-n}\right)
$$

to $Y^{\prime}(T)=\xi(T, Y)$ and to see that the series are divergent. It can be shown that $\gamma$ has the asymptotic expansion $\Gamma$ as $t \rightarrow+\infty$. It follows from results in [3] that given any globally (called "finitely" in [4) subanalytic $S \subseteq \mathbb{R}^{3}$, either the graph of $\gamma$ lies ultimately in $S$ or is ultimately disjoint from $S$. Hence, $\gamma$ is non-oscillatory over $\mathbb{R}_{\mathrm{an}}$. Now, every ultimately nonzero function $f:(a, \infty) \rightarrow \mathbb{R}$ definable in $\mathbb{R}_{\mathrm{an}}$ is ultimately of the form $t^{q} g\left(t^{-1 / d}\right)$ for some $q \in \mathbb{Q}$, positive integer $d$, and analytic 
$g:(-\epsilon, \epsilon) \rightarrow \mathbb{R}$ with $g(0) \neq 0$. Clearly, neither $\gamma_{1}$ nor $\gamma_{2}$ are of this form (since $\gamma \sim \Gamma)$. Hence, no restriction of $\gamma$ to an interval $(a, \infty)$ is definable in $\mathbb{R}_{\mathrm{an}}$.

Let $0 \in A \subseteq \mathbb{R}^{2}$ be subanalytic with no interior. By intersecting $A$ with a sufficiently small open ball about the origin, we reduce to the case that $A \backslash\{0\}$ is a finite union of curves, each definable in $\mathbb{R}_{\mathrm{an}}$. Note that $\gamma(t) \rightarrow 0$ as $t \rightarrow+\infty$ and apply Corollary 2 .

Remark. It can be shown (using the explicit computation of the coefficients of $\Gamma$ ) that $\gamma$ is not definable in many of the known o-minimal structures. It can also be shown that no two distinct such $\gamma$ are definable in any one o-minimal structure. (It would take us too far afield to discuss the details here.)

Proof of Theorem Q (For readers familiar with o-minimal model theory.) Suppose that $\left(\mathfrak{R}_{G}, F\right)$ is o-minimal. Let $\mathcal{H}^{\prime}$ denote the Hardy field of germs at $+\infty$ of unary functions definable in $\left(\mathfrak{R}_{G}, F\right)$. Regard $\left(\mathfrak{R}_{G}, F\right)$ as being expanded by all of its definable functions (including all constants) and a predicate for $<$. Do the same for $\mathfrak{R}_{G}$. Then each of $\mathcal{H}, \mathcal{H}\langle\langle F\rangle\rangle$ and $\mathcal{H}^{\prime}$ can be regarded as elementary extensions of $\mathfrak{R}_{G}$, with $\mathcal{H}$ equal to the definable closure of $x$ over $\mathfrak{R}_{G}$, and $\mathcal{H}\langle\langle F\rangle\rangle$ equal to the definable closure of $F_{1}, \ldots, F_{l}$ over $\mathcal{H}$. (We leave the details to the reader; similar arguments are found in [5] and [7].) Let $K$ be the field of exponents of $\mathfrak{R}_{G}$. The value group $\nu\left(\mathcal{H}\langle\langle F\rangle\rangle^{*}\right)$ is an ordered $K$-linear space, with scalar map $(r, \nu(f)) \mapsto$ $\nu\left(|f|^{r}\right)$. By [5, Theorem C], we have $\operatorname{dim}_{K} \nu\left(\mathcal{H}\langle\langle F\rangle\rangle^{*}\right) \leq l+1$. Hence, $\nu\left(\mathcal{H}\langle\langle F\rangle\rangle^{*}\right)=$ $K . \nu(x)+\sum_{i=1}^{d} K . \nu\left(u_{i}\right)$ for some $d \leq l$ and $0<u_{1}, \ldots, u_{d} \in \mathcal{H}\langle\langle F\rangle$.

Proof of Theorem 3. Let $\mathfrak{R}$ be an o-minimal structure and $f: \mathbb{R} \rightarrow \mathbb{R}$ be definable in the Pfaffian closure $\mathcal{P}(\mathfrak{R})$ of $\mathfrak{R}$. By [13, Corollary 5.2] there exist $a \in \mathbb{R}, n \in \mathbb{N}$, a connected embedded $C^{2}$ submanifold $M \subseteq \mathbb{R}^{2+n}$, a vector field $\xi$ on $M$ definable in $\mathfrak{R}$, and a cell $D$ definable in $\mathcal{P}(\Re)$ of dimension 1 , such that $D$ is a trajectory of $\xi$ and the graph of $f\lceil(a, \infty)$ is equal to the projection of $D$ on the first two coordinates. Since $D$ is a cell, there is a map $F=\left(F_{1}, \ldots, F_{n+1}\right):(a, \infty) \rightarrow \mathbb{R}^{1+n}$, definable in $\mathcal{P}(\mathfrak{R})$, such that $F_{1}=f \uparrow(a, \infty)$ and $D=\operatorname{graph}(F)$. Then for all $t \in$ $(a, \infty)$ the tangent vector to the graph of $F$ at $(t, F(t))$ is tangent to $\xi(t, F(t))$. By reparametrizing, we may assume that the projection of the image $\xi(M)$ on the first variable is identically equal to 1 . By arguing as in the proof of [12 Proposition 4] we obtain $n \in \mathbb{N}, g: \mathbb{R}^{2+n} \rightarrow \mathbb{R}$ definable in $\mathfrak{R}$ and $a \in \mathbb{R}$ such that $f\lceil(a, \infty)$ is $C^{n+1}$ and $f^{(n+1)}(t)=g\left(t, f(t), \ldots, f^{(n)}(t)\right)$ for all $t>a$. Apply Corollary 1 .

\section{Generalizations AND EXtensions}

Exponential comparability. Germs $u, v \in \mathcal{I}$ are exponentially comparable if there exists $n \in \mathbb{N}$ such that $f \leq e_{n} \circ g$ and $g \leq e_{n} \circ f$. One defines exponential comparability classes and the exponential rank of a Hardy field in the obvious way. (See e.g. Kuhlmann and Kuhlmann [11] for more information.) An exponential analog of Lemma 4 is easily obtained. Hence, arguing as for Theorem 1 if $\mathfrak{R}_{G}$ is exponential and exponentially bounded (that is, has exponential rank 1 ) and $F$ is non-oscillatory over $\mathfrak{R}_{G}$, then the exponential rank of $\mathcal{H}\langle\langle F\rangle\rangle$ is at most $l+1$. But we do not yet see how to use this observation to draw conclusions about the growth rates of $F_{1}, \ldots, F_{l}$. (Results are known in some special cases; see [19].)

There is, as yet, no analog of Theorem 2 for exponential rank. 
Other o-minimal structures. The notion of o-minimality makes sense for structures on arbitrary real closed fields; see e.g. [6]. By using results from [18], one easily develops an analog of Hardy field theory for such structures. The obvious modification of Theorem 2 holds, just by replacing "polynomially bounded" with "power bounded". (Power functions are definable homomorphisms of the positive multiplicative group of the underlying ordered field.) Then Corollaries 1 and 2 hold provided that $(R,+, \cdot, G, F)$ is o-minimal, where $R$ is the underlying set of the real closed field under consideration and $F, G$ are defined over $R$. On the other hand, in the paragraph following Corollary 1 the assumption of polynomial bounds must be retained. There are power bounded o-minimal structures that are not polynomially bounded (see e.g. [18, 1.4]) and these structures define unary $C^{\infty}$ functions that vanish precisely on the nonpositive elements of the field. (Take any $\alpha$ in the field of exponents with $\alpha>\mathbb{N}$ and extend $x^{\alpha}$ by $t \mapsto 0$ for $t \leq 0$.)

Although the definition of "non-oscillatory over" extends easily to any ordered structure, it is unclear as to how useful it is. For example, we don't see how to

obtain appropriate modifications of [21, Theorem 3] or [23, Theorem 1] without at least knowing that the mean value theorem holds for unary functions definable in $(R,+, \cdot, G, F)$.

The notion of Pfaffian closure does not make sense, as it stands, when not working over $\mathbb{R}$

\section{REFERENCES}

1. E. Bierstone and P. Milman, Semianalytic and subanalytic sets, Inst. Hautes Études Sci. Publ. Math. 67 (1988), 5-42. MR 89k:32011

2. M. Boshernitzan, Universal formulae and universal differential equations, Ann. of Math. 124 (1986), 273-291. MR 88a:12007

3. F. Cano, R. Moussu, and F. Sanz, Oscillation, spiralement, tourbillonnement, Comment. Math. Helv. 75 (2000), 284-318. MR 2001k:37034

4. L. van den Dries, A generalization of the Tarski-Seidenberg theorem, and some nondefinability results, Bull. Amer. Math. Soc. (N.S) 15 (1986), 189-193. MR 88b:03048

5. _ T-convexity and tame extensions. II, J. Symbolic Logic 62 (1997), 14-34. MR 98h:03048

6. - Tame topology and o-minimal structures, London Math. Soc. Lecture Note Ser., vol. 248, Cambridge University Press, Cambridge, 1998. MR 99j:03001

7. L. van den Dries, D. Marker, and A. Macintyre, The elementary theory of restricted analytic fields with exponentiation, Ann. of Math. 140 (1994), 183-205. MR 95k:12015

8. L. van den Dries and C. Miller, Geometric categories and o-minimal structures, Duke Math. J. 84 (1996), 497-540. MR 97i:32008

9. L. van den Dries and P. Speissegger, The real field with convergent generalized power series, Trans. Amer. Math. Soc. 350 (1998), 4377-4421. MR 99a:03036

10. London Math. Soc. 81 (2000), 513-565.

11. F.-V. Kuhlmann and S. Kuhlmann, The exponential rank of nonarchimedean exponential fields, Real Algebraic Geometry and Ordered Structures, Contemp. Math., vol. 253, Amer. Math. Soc., Providence, RI, 2000, pp. 181-201. MR 2001d:12012

12. J.-M. Lion, Inégalité de Eojasiewicz en géométrie pfaffienne, Illinois J. Math. 44 (2000), 889-900. MR 2001k:32013

13. J.-M. Lion and P. Speissegger, Analytic stratification in the Pfaffian closure of an o-minimal structure, Duke Math. J. 103 (2000), 215-231. MR 2001j:03076

14. D. Marker and C. Miller, Levelled o-minimal structures, Rev. Mat. Complut. (Madrid) 10 (1997), 241-249. MR 99c:03060

15. C. Miller, Expansions of the real field with power functions, Ann. Pure Appl. Logic 68 (1994), 79-94. MR 95i:03081 
16. Exponentiation is hard to avoid, Proc. Amer. Math. Soc. 122 (1994), 257-259. MR 94k:03042

17. _ Infinite differentiability in polynomially bounded o-minimal structures, Proc. Amer. Math. Soc. 123 (1995), 2551-2555. MR 95j:03069

18. _ A growth dichotomy for o-minimal expansions of ordered fields, Logic: From Foundations to Applications, Oxford Sci. Publ., Oxford Univ. Press, New York, 1996, pp. 385-399. MR 98a:03052

19. C. Miller and P. Speissegger, Pfaffian differential equations over exponential o-minimal structures, J. Symbolic Logic 67 (2002), 438-448.

20. J.-P. Rolin, P. Speissegger, and A. Wilkie, Quasianalytic Denjoy-Carleman classes and ominimality, preprint (2001).

21. M. Rosenlicht, The rank of a Hardy field, Trans. Amer. Math. Soc. 280 (1983), 659-671. MR 85d:12002

22. (1984), 829-836. MR 85i:12008

23., Growth properties of functions in Hardy fields, Trans. Amer. Math. Soc. 299 (1987), 261-272. MR 88b:12010

24. J. Shackell, Rosenlicht fields, Trans. Amer. Math. Soc. 335 (1993), 579-595. MR 93d:12012

25. P. Speissegger, The Pfaffian closure of an o-minimal structure, J. Reine Angew. Math. 508 (1999), 189-211. MR 2000j:14093

Laboratoire de Topologie, Université de Bourgogne, 21078 Dijon cedex, France

Current address: IRMAR, Campus Beaulieu, Université Rennes I, 35042 Rennes cedex, France E-mail address: lion@maths.univ-rennes1.fr

Department of Mathematics, The Ohio State University, 231 West 18th Avenue, Columbus, Оhiо 43210

E-mail address: miller@math.ohio-state.edu

$U R L:$ http://www.math.ohio-state.edu/ ${ }^{\sim m i l l e r}$

Department of Mathematics, University of Toronto, Toronto, Ontario, Canada M5S 3G3

Current address: Department of Mathematics, University of Wisconsin, 480 Lincoln Drive, Madison, Wisconsin 53706

E-mail address: speisseg@math.wisc.edu

$U R L:$ http://www.math.wisc.edu/ ${ }^{\sim}$ speisseg 